\title{
RATIONAL SUBSPACES OF INDUCED REPRESENTATIONS AND NILMANIFOLDS
}

BY

R. PENNEY

\begin{abstract}
Recently, Auslander and Brezin developed a technique of distinguishing between certain unitarily equivalent irreducible subspaces of $L^{2}$ of the Heisenberg nilmanifold. In this paper we extend the Auslander-Brezin technique to arbitrary induced representations of arbitrary locally compact groups. We then return to nilmanifolds, showing that the existence of a "nice" theory of distinguished subspaces is equivalent to the existence of square integrable representations for the group.
\end{abstract}

Introduction. In [2], Auslander and Brezin in studying the Heisenberg nilmanifold discovered a technique of distinguishing between certain unitarily equivalent irreducible subspaces of $L^{2}(\Gamma \backslash G)$ on a group theoretical basis. In this paper we develop a technique of distinguishing between certain equivalent subspaces which applies to any induced representation on a locally compact topological group. Our technique is equivalent to the AuslanderBrezin technique for the Heisenberg nilmanifold. Our technique also yields in a large number of cases new realizations of certain irreducible representations on the groups in question and, in the case of nilmanifolds, simplifications in the description of the harmonic analysis of the $L^{2}$ space.

To describe our idea, let $G$ be a locally compact, $\sigma$-compact, separable topological group, and let $K$ be a closed subgroup of $G$. Let $\Omega$ and $\omega$ denote the respective modular functions for $G$ and $K$. Let $U$ be a unitary representation of $K$. We shall adopt the notation $\mathcal{H}(U)$ for the representation space of $U$. Let ind $(K, G, U)$ (or simply ind $(G, U)$ or ind $U$ when context is clear) denote the unitary representation of $G$ induced by $U$, as realized by right translation in the space of $\mathcal{H}(U)$-valued functions $F$ satisfying $F(k x)=$ $(\omega / \Omega)^{1 / 2}(k) V(k) F(x)$ and the usual integrability condition (see [4]). Now if $\tilde{W}$ is a subrepresentation of $U$, then ind $\tilde{W}$ is, in a natural way, a subrepresentation of ind $U$. In fact $\mathcal{H}$ (ind $\tilde{W}$ ) is just the space of functions in $\mathcal{H}$ (ind $U$ ) taking values in $\mathcal{H}(\tilde{W})$. Conversely, whenever $W$ is a subrepresentation of ind $U$ and ind $U$ is realized as above, it makes sense to ask when $\mathcal{H}(W)$ is $\mathcal{H}($ ind $\tilde{W})$ for some subrepresentation $\tilde{W}$ of $U$. This, of course, is a rare

Received by the editors June 27, 1976.

AMS (MOS) subject classifications (1970). Primary 22E25, 22E45, 43A85.

() American Mathematical Society 1979 
circumstance. However, let $L$ be a closed subgroup of $G$ containing $K$. Then $\operatorname{ind}(L, G, \operatorname{ind}(K, L, U))$ is, in a natural way, isomorphic with ind $U$. (The reader may write down the isomorphism between the two realizations if he wishes.) It makes sense to ask, for a given subrepresentation $W$ of ind $U$, does $\mathcal{H}(W)$ equal $\mathcal{H}(\operatorname{ind}(L, G, \tilde{W}))$ for some subrepresentation $\tilde{W}$ of ind $(K, L, U)$. In this case we say that $L$ is $W$ inductive or, equivalently $W$ is $L$ inductive. If $\tilde{W}$ is finite dimensional we say that $W$ is a rational subrepresentation and that $L$ is $W$ rational. In this case we define $\operatorname{dim} \tilde{W}$ to be the rational dimension of $W$.

In $\S 1$ we prove that for any such subrepresentation $W$ there is a unique, minimal, $W$-inductive subgroup $L \supset K$. It is the unique minimal inductive subgroups which serve to distinguish between equivalent subspaces; for clearly $L$ depends on how $W$ sits in ind $U$. From $L$ one can derive many interesting parameters. For example, let $L_{0}$ be the identity component of $L$. Let $A_{0}$ be the algebra of continuous functions on $G$ which are constant on right cosets of $L_{0}$ and let $A$ be the subalgebra of $A_{0}$ consisting of the functions which are constant on right cosets of $L$. Then $A_{0}$ is an $A$ module. If $A_{0}$ is finitely generated over $A$, then the minimal member of generators gives an index which may be used to distinguish between equivalent subspaces. This is essentially the Auslander-Brezin index (see Lemma 1 below). Another possibility is the number of components of $K \backslash L$. In the case of the Heisenberg group this agrees with the index defined above, although it is probably different in general.

In $\S$ II we consider $L^{2}$ of a compact nilmanifold. Specifically, let $G$ be a connected, simply connected nilpotent Lie group and let $\Gamma$ be a uniform subgroup. Then $\Gamma \backslash G$ is compact and has a unique regular $G$ invariant $W$ probability measure $\mu$. We may define a representation $R_{G}$ of $G$ in $L^{2}(\Gamma)$ $G, \mu)$ which acts by right translation. $R_{G}$ is canonically isomorphic with $\operatorname{ind}(\Gamma, G, 1)$ so the above comments apply to subrepresentations of $R_{G}$. We are interested in computing the inductive subgroups for the irreducible subrepresentations of $R_{C}$ and in determining which irreducible subrepresentations are rational. On the rationality question our main result is

THEOREM. Let $W$ be an irreducible subrepresentation of $R_{G}$. W is rational iff $W$ is square integrable modulo its kernel.

The proof leans heavily on the main result of [11] which is essentially the above theorem for maximal primary representations instead of irreducible representations. The maximal primary case is somewhat easier than the above theorem due to the uniqueness of the primary decomposition.

On the question of actually computing the minimal inductive subgroups, our results are less complete. We are only able to treat the so called "constructible" subspaces and these only in the case that there is a normal 
rational subgroup $H$ and an integral character $\chi$ of $H$ which induces a representation equivalent to $W$.

\section{In this section we prove}

THEOREM I. Let notation be as in the introduction. Then there is a unique minimal $W$-inductive subgroup containing $K$.

Proof. Let $K, U, W$, etc. be as above. Let $V=$ ind $U$. Let $\pi$ be the orthogonal projection of $\mathcal{H}(V)$ onto $\mathcal{H}(W)$. Let $A$ be the space of continuous bounded functions on $G$ which are constant on right cosets of $K$. For $f \in A$, let $B(f)$ be the operator on $\mathcal{H}(V)$ given by pointwise multiplication by $f$. (Recall that $\mathcal{H}(V)$ is a space of $\mathcal{H}(U)$ valued functions.) Let $A_{\pi}$ be the set of $f$ in $A$ for which $B(f)$ commutes with $\pi . A_{\pi}$ is a subalgebra of $A$ under the pointwise operations. Let $L$ be the set of $x$ for which $f(x)=f(e)$ for all $f$ in $A_{\pi}$. It is easily verified that $V(x) B(f) V\left(x^{-1}\right)=B(h)$ where $h=f(\cdot x)$. It follows that $A_{\pi}$ is right translation invariant, and hence that $L$ is a closed subgroup of $G$ such that each element of $A_{\pi}$ is constant on right cosets of $L$. We shall show that $L$ is the desired minimal inductive subgroup.

LEMMA I. $A_{\pi}$ is the set of all continuous bounded functions which are constant on right cosets of $L$.

Proof. Let $C B(L \backslash G)$ denote the continuous bounded functions on $L \backslash G$, with the topology of uniform convergence on compacta. Since each element of $A_{\pi}$ is constant on cosets of $L$, we may consider $A_{\pi}$ as a subspace of $C B(L \backslash G)$. It is in fact a closed subspace; for let $f_{n} \rightarrow f$ in $C B(L \backslash G)$, $f_{n} \in A_{\pi}$. Let $g \in \mathcal{H}(V)$ be such that $\|g(\cdot)\|$ has compact support modulo $K$. (There is a dense set of such $g$.) Then $f_{n}$ (now considered as a sequence of functions on $G$ ) converges uniformly on the support of $\|g(\cdot)\|$. Hence $B\left(f_{n}\right) g$ converges in $\mathcal{H}(V)$ to $B(f) g$. Since each $B\left(f_{n}\right)$ commutes with $\pi, B(f)$ commutes with $\pi$ at $g$. Hence, by the density of the $g, B(f)$ commutes with $\pi$. Hence $f$ is in $A_{\pi}$.

Furthermore $A_{\pi}$ is a conjugate-closed subalgebra of $C B(L \backslash G)$ which contains the constant functions and which (by definition) separates points. It follows from the Stone-Weierstrass theorem, the $\sigma$-compactness of $G$ and a Cantor-diagonal argument that $A_{\pi}$ is dense in $C B(L \backslash G)$. This proves the lemma.

COROLlaRY. If $f$ is a bounded measurable function constant on right cosets of $L$, then $B(f)$ commutes with $\pi$.

The fact that $L$ is a minimal inducing subgroup is a consequence of the above corollary and the imprimitivity theorem of Mackey. To see this, let $p$ : $G \rightarrow L \backslash G$ be the projection. For any measurable subset $Y$ of $L \backslash G$, let $f_{Y}$ 
denote the characteristic function of $p^{-1}(Y)$ and let $\phi(Y)=B\left(f_{Y}\right)$. Then $Y \rightarrow \phi(Y)$ is a system of imprimitivity for $V$. It is in fact the system canonically associated with the realization of $V$ as $\operatorname{ind}(L, G,(\operatorname{ind}(K, L, U)))$. Furthermore, the imprimitivity theorem yields a one-to-one correspondence between invariant subspaces of ind $(K, L, U)$ and invariant subspaces of $V$ which are invariant under $\phi$. In fact if $\mathscr{K}_{0}$ is an invariant subspace of $\mathcal{H}(\operatorname{ind}(K, L, U))$, then the space $\mathcal{K}_{1}$ of $\mathcal{K}_{0}$-valued functions in $\mathcal{H}(\operatorname{ind}(L, G$, ind $(K, L, U)))$ is a subspace of $\mathcal{H}(V)$ invariant under $\phi$, and the imprimitivity theorem asserts that these are the only such subspaces. By definition $L$ is $\mathcal{F}_{1}$ inductive. We conclude that any invariant subspace of $V$ which is also invariant under $\phi$ is $L$-inductive. The corollary now implies that $W$ is $L$-inductive.

To see that $L$ is minimal, let $L^{\prime}$ be another $W$ inductive subgroup. Then by the above reasoning, if $\phi^{\prime}$ is the system of imprimitivity for $V$ associated with the realization of $V$ as $\operatorname{ind}\left(L^{\prime}, G, \operatorname{ind}\left(K, L^{\prime}, U\right)\right)$, then $\phi^{\prime}$ leaves $\mathcal{H}(W)$ invariant. Hence $\phi^{\prime}$ commutes with $\pi$. This implies that multiplication by elements of $C B\left(L^{\prime} \backslash G\right)$ commutes with $\pi$ and hence $C B\left(L^{\prime} \backslash G\right) \subset A_{\pi}$. Thus $L^{\prime} \supset L$. This also shows uniqueness. Q.E.D.

II. In this section we compute the minimal inductive subgroups for certain irreducible representations. First a general proposition. Let $G$ be as in $\S I$, and let $K$ and $H$ be closed subgroups with $H$ normal and $K H=G$. Let $U, V, W$, etc. be as in §I. Let $K_{H}=K \cap H, U_{H}=U \mid K_{H}$ and $V_{H}=\operatorname{ind}\left(K_{H}, H, U_{H}\right)$. For the sake of simplicity we shall assume that all of the subgroups defined above are unimodular. Then $V \mid H$ is isomorphic with $V_{H}$, the isomorphism being restriction of functions in $\mathcal{H}(V)$ to $H$. If $f$ is in $\mathcal{H}(V), k \in K, h \in H$, then

$$
V(k) f(h)=f(h k)=U(k) f\left(k^{-1} h k\right) .
$$

It follows that under the above defined isomorphism of $\mathcal{K}(V)$ with $\mathcal{H}\left(V_{H}\right)$, $V \mid K$ is isomorphic with the representation $\tilde{V}$ in $\mathcal{H}\left(V_{H}\right)$ defined by

$$
\tilde{V}(k) f(h)=U(k) f\left(k^{-1} h k\right) .
$$

Let $W_{H}$ be the image of $W \mid H$ in $\mathcal{H}\left(V_{H}\right)$ (i.e. $W_{H}$ is the restriction of $V_{H}$ to the image of $\mathcal{H}(W)$ in $\left.\mathcal{H}\left(V_{H}\right)\right)$. Let $L_{H}$ be the minimal $W_{H}$ inductive subgroup of $H$.

Proposition I. $L_{H}$ is normalized by $K$, and $K L_{H}$ is the minimal $W$ inductive subgroup of $G$.

Proof. We shall use the observation made in the proof of Theorem I that minimal inductive subgroups are "level sets" of certain functions. We shall assume that the notation of the proof of Theorem I is again in force so that, for example, the set $A_{\pi}$ is the set of continuous bounded functions $f$ on $G$ 
which are constant on cosets of $K$ in $G$ for which the pointwise multiplication operators $B(f)$ in $\mathcal{H}(V)$ commute with the projection $\pi$ onto $\mathcal{H}(W)$. We shall denote the corresponding entities on $H$ defined relative to $\mathcal{H}\left(W_{H}\right)$ and $K \cap H$ by affixing the subscript $H$. Thus, for example, $B_{H}(f)$ is a pointwise multiplication operator on $\mathcal{H}\left(V_{H}\right)$ defined by a function constant on cosets of $K_{H}$ in $H$.

Now, to show that $L_{H}$ is normalized by $K$ recall that in the proof of Theorem I we showed that $L_{H}$ is the set of $x$ in $H$ such that $f(x)=f(e)$ for all $f$ in $A_{\pi, H}$. In order to show that $H$ is normalized by $K$, it suffices to show that $f \in A_{\pi, H}$ implies that $f_{k}=f\left(k \cdot k^{-1}\right)$ also belongs to $A_{\pi, H}$ for all $k \in K$. This follows from the easily verified fact that

$$
B_{H}\left(f_{k}\right)=\tilde{V}(k) B_{H}(f) \tilde{V}\left(k^{-1}\right) .
$$

Note that $\tilde{V}$ commutes with $\pi_{H}$ since $\tilde{V}$ is isomorphic with $V$ and $\pi_{H}$ is isomorphic with $\pi$.

To show that $L=K L_{H}$, we shall show that the restriction map from functions on $G$ to functions on $H$ maps $A_{\pi}$ onto $A_{\pi, H}$. It will follow that $L_{H}=L \cap H$. But since $L \supset K$ and $K H=G$, we have $L=K(L \cap H)=$ $K L_{H}$, as desired. To prove the statement about restriction, note that if $f \in A$ (the continuous, bounded functions on $G$ constant on cosets of $K$ ) then under the isomorphism of $\mathcal{H}(W)$ with $\mathcal{H}\left(W_{H}\right), B(f)$ is isomorphic with $B_{H}(f \mid H)$. It follows that $B(f)$ commutes with $\pi$ iff $B_{H}(f \mid H)$ commutes with $\pi_{H}$. Hence $f \in A_{\pi}$ iff $f \mid H \in A_{\pi, H}$. Since restriction obviously maps $A$ onto $A_{H}$, we are done. Q.E.D.

Another rather obvious comment which is often useful is the following: Suppose $L^{\prime} \supset K$ is $W$ inductive but not necessarily minimal. Then, as seen above, there is a well-defined subrepresentation $W^{\prime}$ of $\operatorname{ind}\left(K, L^{\prime}, U\right)$ which induces $W$. Then

Proposition II. The minimal $W$ inductive subgroup equals the minimal $W^{\prime}$ inductive subgroup.

The proof of this is similar to the proof of Proposition I and will be omitted.

EXAMPLE. Suppose that $K$ is normal in $G$ and that $K \backslash G$ is compact. Let $U$ be the trivial representation of $K$ in $\mathrm{C}$ and let $V=\operatorname{ind}(K, G, U)$. Then any character $\chi$ of $G$ which is trivial on $K$ belongs to $\mathcal{H}(V)$. Let $W$ be a subrepresentation of $V$ for which $\mathcal{H}(W)$ has an orthonormal basis $S=$ $\left\{\chi_{\alpha}\right\}_{\alpha \in \Lambda}$ of characters of $G$ trivial on $K$. We shall compute the minimal $W$ inductive subgroup $L$. Specifically, let $G^{*}$ be the character group of $G$. Let $S_{0}$ be the set of $\lambda$ in $G^{*}$ such that $\lambda \cdot S=S$. We shall show

Proposition III. $L=\cap_{\lambda} \operatorname{ker} \lambda$ where $\lambda$ ranges over $S_{0}$. The $W$ inductive subspace of $\operatorname{ind}(K, L, 1)$ is the closed subspace spanned by the set $\left\{\chi_{\alpha} \mid L\right\}$ where 
$\alpha$ ranges over $\Lambda$. The rational dimension of $W$ is the number of $S_{0}$ orbits in $S$. (Note that $S_{0}$ is a subgroup of $G^{*}$.)

Proof. First note that since $K$ is normal, it suffices to consider the case where $K=\{e\}$; for $K$ is in the kernel of $V$, so $V$ projects to a representation $V^{\prime}$ on $K \backslash G=G^{\prime}\left(V^{\prime}\right.$ is the right regular representation of $\left.G^{\prime}\right)$; $W$ projects to a subrepresentation $W^{\prime}$ of $V^{\prime}$; and if $L^{\prime}$ is the minimal $W^{\prime}$ inductive subgroup, then the minimal $W$ inductive subgroup is the pull-back of $L^{\prime}$ in $G$.

Now, let $\pi$ be the projection onto $\mathcal{H}(W)$. Let $f$ be a continuous function on $G$ such that the operator $B(f)$ commutes with $\pi$. Then for each $\alpha \in \Lambda, B(f) \chi_{\alpha}$ $\in \mathcal{H}(W)$, so

$$
f \chi_{\alpha}=\sum c_{\alpha, \beta} \chi_{\beta} \quad(\beta \in \Lambda)
$$

where the sum converges in $L^{2}(G)$. Hence $f=\Sigma c_{\alpha, \beta} \chi_{\beta} \bar{\chi}_{\alpha}$. In particular, $f$ has an expression

$$
f=\sum c_{\lambda} \lambda \quad(\lambda \text { a character of } G) .
$$

Furthermore, from the uniqueness of Fourier expansion, if $c_{\lambda} \neq 0$, then for each $\alpha$ there is a $\beta$ such that $c_{\lambda}=c_{\alpha, \beta}$ and $\lambda \chi_{\alpha}=\chi_{\beta}$, i.e. $\lambda \cdot S \subset S$. Also, if we set $\mathcal{K}_{0}$ to be the span of the characters of $G$ in $\mathcal{H}(V)$, then the above expression for $f$ implies that $B(f)$ leaves $\mathcal{K}_{0}$ invariant. $B(f)$ commutes with $I-\pi$, so $B(f)$ leaves $\mathcal{K}(W)^{\perp} \cap \mathcal{K}_{0}$ invariant. This space is spanned by the complement of $S$ in the character group $G^{*}$ of $G$. Reasoning as above, one sees that if $c_{\lambda} \neq 0$, then $\lambda \cdot\left(G^{*} \sim S\right) \subset G^{*} \sim S$. Hence we have $\lambda \cdot S=S$.

Conversely, if $\lambda \in G^{*}$ is such that $\lambda \cdot S=S$, then $B(\lambda)$ leaves $\mathcal{H}(W)$ and $\mathcal{H}(W)^{\perp} \cap \mathcal{H}_{0}$ invariant. Also $B(\lambda)$ leaves $\mathcal{H}_{0}$ and $\mathcal{H}_{0}^{\perp}$ invariant. Hence $B(\lambda)$ leaves $\mathcal{H}(W)^{\perp} \cap \mathcal{K}_{0}+\mathcal{H}_{0}^{\perp}=\mathscr{H}(W)^{\perp}$ invariant, and thus $B(\lambda)$ commutes with $\pi$. It follows that $B(f)$ commutes with $\pi$ iff $f$ has an expression in $L^{2}(G), f=\Sigma c_{\lambda} \lambda\left(\lambda \in S_{0}\right)$. From the proof of Theorem I, $L$ is the set of $x$ for which $f(x)=f(e)$ for all such $f$. This is clearly

$$
L=\cap \operatorname{ker} \lambda \quad\left(\lambda \in S_{0}\right) .
$$

The subrepresentation $\tilde{W}$ of $\operatorname{ind}(K, L, U)=\tilde{V}$ which induces $W$ is also computable. In fact, let $W_{0}$ be the subrepresentation of $\operatorname{ind}(L, G, \tilde{V})=V_{0}$ corresponding to $W$ under the natural isomorphism of $V$ with $V_{0}$. Then $W_{0}=\operatorname{ind}(L, G, \tilde{W})$, so $\mathcal{H}\left(W_{0}\right)$ is the space of functions in $\mathcal{H}\left(V_{0}\right)$ taking values in $\mathcal{H}(\tilde{W})$. It follows that $\mathcal{H}(\tilde{W})=\left\{f(e) \mid f \in \mathcal{H}\left(W_{0}\right), f\right.$ continuous $\}$. Now if $f \in \mathcal{H}(V)$, we may define a mapping $f^{\prime}$ of $G$ into $\mathcal{H}(\tilde{V})$ by $f^{\prime}(x)=f(\cdot x) \mid L$. This belongs to $\mathcal{H}(\tilde{V})$ for a.e. $x$, and $f \rightarrow f^{\prime}$ defines the isomorphism of $\mathcal{H}(V)$ to $\mathcal{K}(\tilde{V})$. Since $\mathcal{H}(W)$ is spanned by the $\chi_{\alpha}, \alpha \in \Lambda$, it follows that $\mathcal{H}\left(W_{0}\right)$ is spanned by the $\left(\chi_{\alpha}\right)^{\prime}$ and hence, that $\mathcal{H}(\tilde{W})$ is spanned by the $\left(\chi_{\alpha}\right)^{\prime}(e)=\chi_{\alpha} \mid L$, as desired.

Note that if we let $L^{\perp}=\left\{\lambda \in G^{*}|\lambda| L \equiv 1\right\}$, then $L^{\perp}=S_{0}$ and $\chi_{\alpha} \mid L=$ 
$\chi_{\beta} \mid L \Leftrightarrow \chi_{\alpha} S_{0}=\chi_{\beta} S_{0}$. Hence the dimension of $\tilde{W}$ is the number of $S_{0}$ orbits in S. Q.E.D.

Now we turn our attention to nilmanifolds. Let $G$ be a connected, simply connected, nilpotent Lie group. Let $\Gamma$ be a closed discrete subgroup for which $\Gamma \backslash G$ is compact. Then $\Gamma \backslash G$ has an invariant measure. Let $R_{G}$ denote the right regular representation of $G$ in $L^{2}(\Gamma \backslash G)$. Note that $R_{G}=\operatorname{ind}(\Gamma, G, 1)$. Let $U$ be an irreducible representation of $G$ occurring in $R_{G}$. Let $W$ be an irreducible subrepresentation of $R_{G}$ which is equivalent to $U$. Let $L$ be the minimal $W$-inductive subgroup of $G$ containing $\Gamma$ and let $W_{L}$ be the corresponding subrepresentation of ind $(\Gamma, L, 1)=R_{L}$. We shall prove the following theorem:

THEOREM II. $W_{L}$ is finite dimensional iff $U$ is square integrable modulo its kernel.

The term "square integrable modulo its kernel" requires some comments. Let $I$ denote the identity operator and let $K=\{g \in G \mid U(g)=c(g) I$ for some $c(g) \in \mathbf{C}\}$. Then for any $v, w \in \mathcal{H}(U), g \rightarrow|(U(g) v, w)|$ is constant on cosets of $K . U$ is said to be square integrable modulo its kernel iff there is a choice of $v$ and $w$ for which the above function is nonzero and square integrable on $K \backslash G$.

Proof. We shall first show that rationality implies square-integrability. To this end let $\pi_{0}$ be the projection onto the $U$ primary subspace of $R_{G}$. We shall apply the main result of [11] which states that $W$ is square integrable modulo its kernel iff $\pi_{0}$ maps $C(\Gamma \backslash G)$ into $C(\Gamma \backslash G)$. It is known [10] that $\pi_{0}$ preserves $C(\Gamma \backslash G)$ iff the projection $\pi$ onto $\mathcal{H}(W)$ preserves $C(\Gamma \backslash G)$. We shall sketch a proof of this fact in a moment. In any event, it suffices then to show that $\pi$ preserves $C(\Gamma \backslash N)$. But $\pi$ can be expressed in terms of the projection $\pi_{L}$ onto $\mathcal{H}\left(W_{L}\right)$ in $L^{2}(\Gamma \backslash L)$. To see this it will be convenient to consider $R_{L}$ and $R_{G}$ as induced representations so $\mathcal{H}\left(R_{L}\right)$ and $\mathcal{H}\left(R_{G}\right)$ are thought of as spaces of functions on $L$ and $G$, respectively. For $f \in \mathcal{H}\left(R_{G}\right)$ let $f^{\prime}: G \rightarrow \mathcal{H}\left(R_{L}\right)$ be defined by

$$
f^{\prime}(x)=R(x) f \mid L .
$$

The mapping $f \rightarrow f^{\prime}$ defines the isomorphism of $R_{G}$ onto ind $\left(L, G, R_{L}\right)$. The inverse mapping is given by

$$
f(x)=f^{\prime}(x)(e) .
$$

The projection $\pi^{\prime}$ onto the subspace of $\mathscr{H}\left(\operatorname{ind}\left(L, G, R_{L}\right)\right)$ induced from $\mathcal{H}\left(W_{L}\right)$ is given by

$$
\left(\pi^{\prime} g\right)(x)=\pi_{L}(g(x))
$$

for any $g \in \mathcal{H}\left(\operatorname{ind}\left(L, G, R_{L}\right)\right)$. Since $\pi$ is isomorphic with $\pi_{L}$ under $f \rightarrow f^{\prime}$, it 
follows that

$$
(\pi f)(x)=\pi_{L}\left(f^{\prime}(x)\right)(e) .
$$

Since $\pi_{L}$ has finite dimensional range, its range consists entirely of continuous functions. Hence $\pi_{L}$ maps $C(\Gamma \backslash L)$ into $C(\Gamma \backslash L)$ and $\left\|\pi_{L}(f)\right\|_{\infty} \leqslant C\|f\|_{\infty}$ for some constant $C$ and all continuous $f$. Hence from the above formula for $\pi$,

$$
|\pi(f)(x)|=\left|\pi_{L}\left(f^{\prime}(x)\right)(e)\right| \leqslant C\left\|f^{\prime}(x)\right\|_{\infty} \leqslant C\|f\|_{\infty} .
$$

Thus $\pi$ preserves $C(\Gamma \backslash G)$, as desired.

To finish the first part of our theorem we should, as promised, sketch the proof of the fact that the preservation of $C(\Gamma \backslash G)$ under $\pi$ is equivalent to the preservation of $C(\Gamma \backslash G)$ under $\pi_{0}$. This involves an analysis of the intertwining algebra of $R_{G}$.

Let $x \in G$ be such that $\Gamma_{x}=x \Gamma x^{-1} \cap \Gamma$ has finite index in $\Gamma$. If $f$ is a function on $\Gamma \backslash G$, then the function $g$ defined by $g(y)=f\left(\Gamma x^{-1} y\right)$ satisfies $g(\gamma y)=g(y)$ for $\gamma \in \Gamma_{x}$. Let

$$
M(x) f(\Gamma y)=\left[\Gamma_{x}: \Gamma\right]^{-1} \sum g(\gamma y) \quad\left(\gamma \in \Gamma_{x} \backslash \Gamma\right) .
$$

Then the operators $M(x)$ map $L^{2}(\Gamma \backslash G)$ into $L^{2}(\Gamma \backslash G)$ and intertwine $R_{G}$. In fact, the algebra generated by the $M(x)$ is the Hecke algebra of $\Gamma \backslash G$. It is a well-known consequence of Moore's multiplicity one theorem [8] that this algebra is weakly dense in $R_{G}$ 's intertwining algebra. We may, in fact, restrict to rational $x$ in $G$. For another proof of this fact, see [10].

Now let $\sigma$ be another projection onto another irreducible subspace of $R_{G}$. Suppose that the restriction of $R_{G}$ to the image of $\sigma$ is equivalent to $W$. There exist elements $A$ and $B$ in the intertwining algebra of $R_{G}$ such that $A \pi B=\sigma$. In fact there is a unitary map $T$ from the image of $\sigma$ to the image of $\pi$ which intertwines $R \mid \sigma$ and $R \mid \pi$. Let $B=T \sigma$ and let $A=T^{-1} \pi$. Now the operators $A \pi B$ with $A$ and $B$ in $R_{G}$ 's intertwining algebra map $\mathcal{H}\left(R_{G}\right)$ onto an $R_{G}$ invariant subspace which, if not zero, transforms according to $W$. Since $W$ occurs with only finite multiplicity in $R_{G}$, the set of such operators spans a finite dimensional space. If $A$ and $B$ range over the Hecke algebra, the corresponding set of operators forms a dense subspace of a finite dimensional space-i.e. the whole space. Hence $\sigma=A \pi B$ with $A$ and $B$ in the Hecke algebra. It follows that $\sigma$ maps $C(\Gamma \backslash G)$ into $C(\Gamma \backslash G)$. Hence the projection $\pi_{0}$ onto the $W$-primary subspace of $R$, being a finite sum of such $\sigma$ 's, also preserves $C(\Gamma \backslash G)$. By similar reasoning $\pi=D \pi_{0}$ for some $D$ in the Hecke algebra so $\pi$ will preserve $C(\Gamma \backslash G)$ if $\pi_{0}$ does. This completes the first part of our proof.

To see the converse, suppose $W$ is square integrable modulo its kernel. Let $\pi_{0}$ be the projection onto the $W$ primary subspace of $R_{G}$. Then it is not difficult to show from [9] (see [11, Theorem 1, (i) $\Rightarrow$ (iv)]) that there is a closed 
subgroup $H \supset \Gamma$ and a character $\lambda$ of $H$ such that

$$
\pi_{0} f(\Gamma x)=\int_{\Gamma \backslash H} f(\Gamma h x) \lambda(h) d h .
$$

$H$ is, in fact, $\Gamma K$ and $\lambda$ is defined by $\lambda(\gamma k)=c(k)$ where $K$ and $c$ are as above. Let $\pi$ be the projection onto $\mathcal{K}(W)$. It follows from the finite dimensionality of the intertwining algebra, that there is an element $A$ of the Hecke algebra such that $A \pi_{0}=\pi$. In fact, we can take

$$
A=\sum_{i=1}^{n} c_{i} M\left(x_{i}\right)
$$

where the $x_{i}$ are rational. Note that for some $C \in \mathbf{C}$,

$$
M(x) M(y)=C \sum M(x \gamma y) \quad\left(\gamma \in \Gamma_{x^{-1}} \backslash \Gamma\right),
$$

so that the Hecke algebra is, in fact, spanned by the operators $M(x)$.

Let $L$ be the subgroup generated by $H$ and the set $\left\{x_{1}, \ldots, x_{n}\right\}$.

LEMMA. $L$ is closed.

Proof. Let $H_{0}$ be the identity component of $H$. $\Gamma H_{0}$ is open in $H$ and hence is closed. In $H_{0} \backslash G, H_{0} \backslash \Gamma H_{0}$ is uniform. Hence $H_{0} \backslash H$ is rationally generated relative to the rational structure on $H_{0} \backslash G$ defined by $H_{0} \backslash \Gamma H_{0}$ (see [1]). Thus $\left\{H_{0} x_{i}\right\}$ and $H_{0} \backslash H$ generate a discrete subgroup of $H_{0} \backslash H$ since $\left\{H_{0} x_{i}\right\}$ are also rational. $L$ is the pull-back of this subgroup. Q.E.D.

Now in the space of $\operatorname{ind}(\Gamma, L, 1)=R_{L}$ we may define for any rational point $x \in L$ an operator $M_{L}(x)$ by the same formula that $M(x)$ was defined by above. Let $A_{L}=\Sigma c_{i} M_{L}\left(x_{i}\right)$ where the $c_{i}$ and $x_{i}$ are the same as in (*) above. Let $\pi_{0, L}$ be defined as in ( $\dagger$ ) relative to $L$ and let $\pi_{L}=A_{L} \pi_{0, L}$. Let $W_{L}$ be the subrepresentation of $R_{L}$ obtained from restricting $R_{L}$ to the image of $\pi_{L}$.

LEMMA. $L$ is $W$ inductive and $W_{L}$ is the corresponding subrepresentation of $R_{L}$.

Proof. If $f \in C(\Gamma \backslash G)$,

$$
M\left(x_{i}\right) f(\Gamma e)=M_{L}\left(x_{i}\right) \tilde{f}(\Gamma e),
$$

where $\tilde{f}=f \mid \Gamma \backslash L$. Hence

$$
M\left(x_{i}\right) f(\Gamma x)=M_{L}\left(x_{i}\right)\left(R_{G}(x) f\right)^{\sim}(\Gamma e) .
$$

It follows that

$$
(\pi f)(\Gamma x)=\pi_{L}\left(R_{G}(x) f\right)^{\sim}(\Gamma e) .
$$

If $x \in L$, the right-hand side of the above equality becomes $\pi_{L} \tilde{f}(\Gamma x)$ so we conclude that $(\pi f)^{\sim}=\pi_{L} \tilde{f}$. If $\pi f=f$, it follows that $(R(x) f)^{\sim} \in \mathcal{H}\left(W_{L}\right)$ for all $x$. The map $f \rightarrow(R(x) f)^{\sim}$ defines the isomorphism of $R_{G}$ with 
$\operatorname{ind}\left(L, G, R_{L}\right)$. Thus $f \in \mathcal{H}\left(\operatorname{ind}\left(L, G, W_{L}\right)\right)$.

To see the converse inclusion, note that $\pi_{L}$ is a projection, since for all $f \in C(\Gamma \backslash G)$,

$$
\pi_{L}^{2}\left((R(x) f)^{\sim}\right)=\pi_{L}\left((R(x) \pi f)^{\sim}\right)=\left(R(x) \pi^{2} f\right)^{\sim}=\pi_{L}(R(x) f)^{\sim} .
$$

Hence if $f \in \mathcal{K}$ (ind $\left.W_{L}\right)$,

$$
(\pi(R(x)) f)^{\sim}=\pi_{L}(R(x) f)^{\sim}=R(x) \tilde{f},
$$

and hence $\pi(f)=f$. This proves the lemma.

To prove the theorem note that $W_{L}$ is a subrepresentation of $\operatorname{ind}(H, L, \lambda)$ and $H \backslash L$ is finite, so $W_{L}$ is finite dimensional. Hence $W$ is rational. Q.E.D.

COROLlaRY. Let $W$ be an irreducible subrepresentation of $R_{G}$ corresponding to a representation $U$ which is square integrable modulo its kernel. Let $L^{\prime}$ be the minimal $W$ inductive subgroup. Then $L_{0}^{\prime}=K=\{g \in G \mid U(g)=c(g) I\}$.

Proof. From the proof of the above theorem and the fact that the subgroup $H$ constructed in [11] is $\Gamma K$, it follows that $L^{\prime} \subset L$ and hence that $L_{0}^{\prime} \subset L_{0}=H_{0}=K$. On the other hand, $R_{G} \mid W$ is scalar on $K$ so $K \subset L_{0}^{\prime}$. Q.E.D.

The above corollary says that $L_{0}^{\prime}$ does not depend on $W$. In this case $\Gamma L_{0}^{\prime} \backslash L^{\prime}$ is finite. $\left[\Gamma L_{0}^{\prime}: L^{\prime}\right]$ is the natural analogue of the Auslander-Brezin index in this case.

The above theorem is not very useful for actually computing the minimal inductive subgroups, for expressing $\pi$ as a linear combination of $M\left(x_{i}\right) \sigma$ is difficult. Using the theory developed in the previous section, we can obtain a computational method which is valid whenever $W$ is inducible from a suitable normal subgroup of $G$. Specifically, it is known that there is a closed subgroup $H$ of $G$ and a character $\chi$ of $H$ such that $W$ is equivalent to $\operatorname{ind}(H, G, \chi)=U^{x}$. Furthermore $H$ may be chosen so that $\Gamma H$ is closed and $\chi$ is trivial on $H \cap \Gamma$ (see [6] or [12]). Let us suppose also that $H$ is normal. We may extend $\chi$ to a function $\tilde{\chi}$ on $\Gamma H$ by setting $\tilde{\chi}(\gamma h)=\chi(h)$. $(\tilde{\chi}$, of course, is not usually a character). However, $\tilde{\chi}$ belongs to $\mathcal{H}(\operatorname{ind}(\Gamma, \Gamma H, 1))$.

If $\gamma \in \Gamma$, the function $\chi_{\gamma}=\chi\left(\gamma \cdot \gamma^{-1}\right)$ is a character of $H$ trivial on $H \cap \Gamma$ so we may form $\left(\chi_{\gamma}\right)^{\sim}$ as above. The closure of the span of the $\left(\chi_{\gamma}\right)^{\sim}$ in $\mathcal{H}\left(R_{\Gamma H}\right)$ is $R_{\Gamma H}$ invariant and hence restriction of $R_{\Gamma H}$ to this subspace defines a subrepresentation $W_{H}$.

LEMMA. $W_{H} \approx \operatorname{ind}(H, \Gamma H, \chi)$.

Proof. Since $U^{x}$ is irreducible, $\chi_{\gamma}=\chi$ iff $\gamma \in H$. Furthermore, the $\left(\chi_{\gamma}\right)^{\sim}$ 
are orthonormal in $\mathcal{H C}\left(R_{\gamma}\right)$. If $f \in \mathcal{H}(\operatorname{ind}(H, \Gamma H, \chi))$, let

$$
T f=\sum_{\Gamma \cap H \backslash \Gamma} f(\gamma)\left(\chi_{\gamma}\right)^{\sim} .
$$

The sum converges in $\mathcal{H}\left(W_{H}\right)$, since by definition of the induced representation $\|f\|^{2}=\Sigma|f(\gamma)|^{2}$. Clearly $T$ defines a unitary isomorphism of representations. Q.E.D.

It follows that ind $W_{H} \approx U^{x}$, so ind $W_{H}$ is irreducible and defines an irreducible subrepresentation $W$ of $R_{G}=$ ind $R_{\Gamma H}$. We shall call $W$ the constructible subrepresentation defined by $(H, \chi)$. It is our goal to compute the minimal $W$ inductive subgroup.

From Proposition II, the minimal $W$ inductive subgroup $L$ is the minimal $W_{H}$ inductive subgroup. Proposition $I$ applies to $W_{H}$, with $\Gamma$ in place of $K$. Specifically, we identify $R_{\Gamma H} \mid H$ with $R_{H}=\operatorname{ind}(\Gamma \cap H, H, 1)$. Then $\mathcal{H}\left(W_{H}\right)$ is identified with the space spanned by the $\chi_{y}$ in $\mathcal{H}\left(R_{H}\right)$. Proposition I says that $L=\Gamma L^{\prime}$ where $L^{\prime}$ is the minimal $W_{H} \mid H$ inductive subgroup of $H . L^{\prime}$ can be computed from the above example. Our result is

THEOREM III. Let $S=\left\{\chi_{\gamma} \mid \gamma \in \Gamma\right\}$. Let $S_{0}$ be the set of characters $\lambda$ of $H$ such that $\lambda \cdot S=S$. Let $L^{\prime}=\cap_{\lambda} \operatorname{ker} \lambda$ where $\lambda$ ranges over $S_{0}$. Then the minimal $W$ inductive subgroup is $L=\Gamma L^{\prime}$. The rational dimension of $W$ is the number of $S_{0}$ orbits in $S$.

From the above theorem we may obtain a sharpening of a result of Brezin [3] and Corwin and Greenleaf [5].

Corollary. $\operatorname{ind}(H, G, \chi)$ is square integrable modulo its kernel iff $\left\{\chi_{\gamma} \mid \gamma \in\right.$ $\Gamma\}$ is a finite union of cosets of a single subgroup $S_{0}$ in the group of characters of $H$ trivial on $\Gamma \cap H$.

\section{REFERENCES}

1. L. Auslander, An exposition of the structure of solvmanifolds. I, Bull. Amer. Math. Soc. 79 (1973), 227-261.

2. L. Auslander and J. Brezin, Invariant subspace theory for three-dimensional nilmanifolds, Bull. Amer. Math. Soc. 78 (1972), 255-258.

3. J. Brezin, Note on harmonic analysis on nilmanifolds, Proc. Conf. (Marseilles-Luminy, August 1974) (to appear).

4. R. J. Blattner, On induced representations, Amer. J. Math. 83 (1961), 79-98. MR 23 \#A2757.

5. L. Corwin and F. Greenleaf, Integral formulas with distribution kernels for irreducible projections in $L^{2}$ of a nilmanifold (preprint).

6. R. Howe, On Frobenius reciprocity for unipotent algebraic groups over $Q$, Amer. J. Math. 93 (1971), 163-172. MR 43 \#7556.

7. G. W. Mackey, The theory of group representations, Lecture notes by Dr. Fell and Dr. Lowdenslager, Univ. of Chicago, Chicago, Ill., 1955. MR 19, 117.

8. C. C. Moore, Decomposition of unitary representations defined by discrete subgroups of nilpotent groups, Ann. of Math. (2) 82 (1965), 146-182. MR 31 \#5928. 
9. C. C. Moore and J. A. Wolf, Square integrable representations of nilpotent groups, Trans. Amer. Math. Soc. 185 (1973), 445-462. MR 49 \#3033.

10. R. Penney, The intertwining algebra of $L^{2}$ of a nilmanifold (preprint).

11.

12. L. F. Richardson, Decomposition of the $L^{2}$-space of a general compact nilmanifold, Amer. J. Math. 93 (1971), 173-190. MR 44 \#1771.

Department of Mathematics, PURdue University, West Lafayette, Induna 47907 